\title{
Fabrication and Two-Way Deformation of Shape Memory Composite with SMA and SMP
}

\author{
H. Tobushi ${ }^{1, a}$, S. Hayashi, b , Y. Sugimoto ${ }^{1, c}$ and K. Date ${ }^{1, c}$ \\ ${ }^{1}$ Department of Mechanical Engineering, Aichi Institute of Technology \\ 1247 Yachigusa, Yakusa-cho, Toyota, 470-0392, Japan. \\ ${ }^{2}$ SMP Technologies Inc., Shendagaya 3-50-11, Shibuyaku, Tokyo, 151-0051, Japan \\ atobushi@aitech.ac.jp, ${ }^{b}$ hayashi@smptechno.com, 'tobulabo@aitech.ac.jp
}

Keywords: Shape memory alloy, Shape memory polymer, Shape memory effect, Superelasticity, Composite, Two-way, Bending, Recovery force

\begin{abstract}
The shape memory composite (SMC) with shape memory alloy (SMA) and shape memory polymer (SMP) was fabricated, and the two-way bending deformation and recovery force were investigated. The results obtained can be summarized as follows. (1) Two kinds of SMA tapes which show the shape memory effect and superelasticity were heat-treated to memorize the round shape, respectively. The shape-memorized round SMA tapes were sandwiched between the SMP sheets, and the SMC belt was fabricated. (2) The two-way bending deformation with an angle of 56 degrees is observed during heating and cooling. (3) If the SMC belt is heated and cooled by keeping the form, recovery force increases during heating and decreases during cooling.
\end{abstract}

\section{Introduction}

One of the main materials in the intelligent materials is shape memory alloy (SMA) ${ }^{1)}$. Shape memory polymer (SMP) has been also practically used ${ }^{2}$.

In SMAs, the shape memory property appears based on the martensitic transformation (MT) in which the crystal structure varies depending on the variation in temperature. In SMAs, strain of $8 \%$ is recoverable and high recovery stress can be used.

In SMPs, the shape memory property appears based on the glass transition in which the characteristics of molecular motion vary depending on the variation in temperature. In SMPs, sheet, film, foam and other forms can be used and strain of several hundred percents is recoverable.

In order to use new and higher function by combining the excellent qualities of both SMA and SMP, the development of shape memory composite (SMC) with SMA and SMP is expected. If SMP is used as the matrix in SMC and SMA as the fiber, the following properties can be obtained in SMC. (1) Large recovery force appears and (2) the deformed shape is recovered at high temperature, and (3) the deformed shape is held and (4) large load can be carried at low temperature. By combining SMA and SMP, the SMC element can be developed ${ }^{3)}$.

In the present paper, fabrication and mechanical properties of the SMC which shows the two-way deformation depending temperature variation are investigated. With respect to the characteristics of the SMC belt, the bending deformation and the recovery force with the two-way property are discussed.

\section{Characteristics of SMC with SMA and SMP}

In order to discuss the basic deformation properties of SMA and SMP and the characteristics of SMC, the dependence of elastic modulus and yield stress of SMA, SMP and steel on temperature is shown in Figs. 1 (a) and (b), respectively. Figure 1 is expressed on the semi-logarithmic graph. 
In Fig. 1, $A_{s}, A_{f}$ and $T_{g}$ on the temperature axis denote the reverse (austenite) transformation start and finish temperatures of SMA and the glass transition temperature of SMP, respectively. The symbols $\sigma_{M}$ and $\sigma_{A}$ represent the MT stress and reverse transformation stress, respectively.

As shown in Fig. 1, elastic modulus and $\sigma_{M}$ are small at temperatures below $A_{s}$ and large above $A_{f}$ in SMAs. The stress $\sigma_{A}$ appears above $A_{f}$. Both $\sigma_{M}$ and $\sigma_{A}$ increase in proportion to temperature. Based on these characteristics, if SMAs are deformed below $A_{f}$, residual strain appears after unloading and the residual strain disappears by heating under no load, showing the shape memory effect (SME). If SMAs are deformed above $A_{f}$, strain is recovered during unloading, showing superelasticity (SE). On the other hand, elastic modulus and yield stress of steels are almost constant in the temperature range at which most SMAs have been practically used. Therefore, if steel is used as a bias element in combination with SMA elements in the temperature region above and below $A_{f}$, the two-way shape memory effect (TWSME) can be achieved by heating and cooling.

On the other hand, elastic modulus and yield stress of SMPs are large at temperatures below the glass transition temperature $T_{g}$ and small above $T_{g}$. Therefore, SMPs are easily deformed above $T_{g}$. If SMPs are cooled until the temperature below $T_{g}$ by holding the deformed shape constant, the deformed shape is fixed and SMPs can carry large load. This property is called the shape fixity (SF). If the shape-fixed SMP element is heated until the temperature above $T_{g}$ under no load, the original shape is recovered. This property is called the shape recovery (SR).

As mentioned above, the dependence of elastic modulus and yield stress on temperature is quite different among SMA, SMP and steel. Therefore, if the composite material is produced by combining these materials appropriately, the SMC which has new functions can be developed.

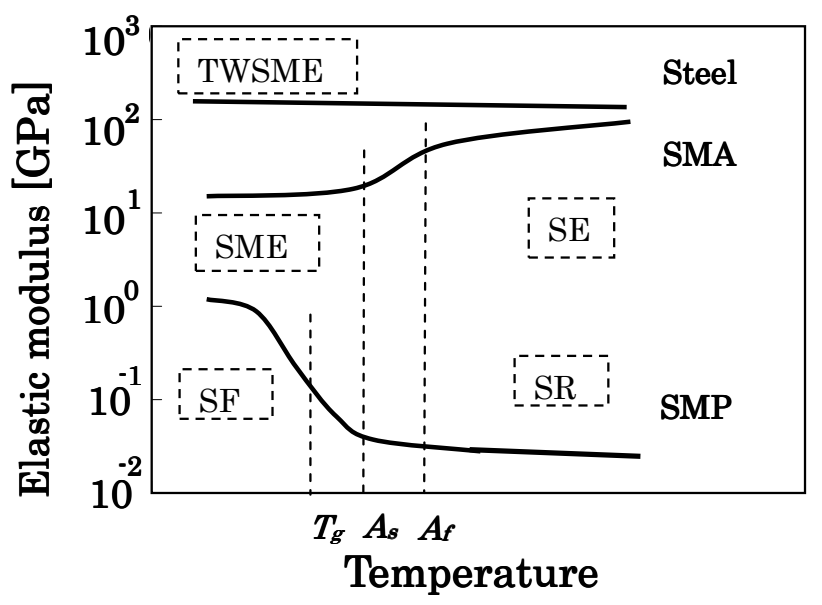

(a) Elastic modulus

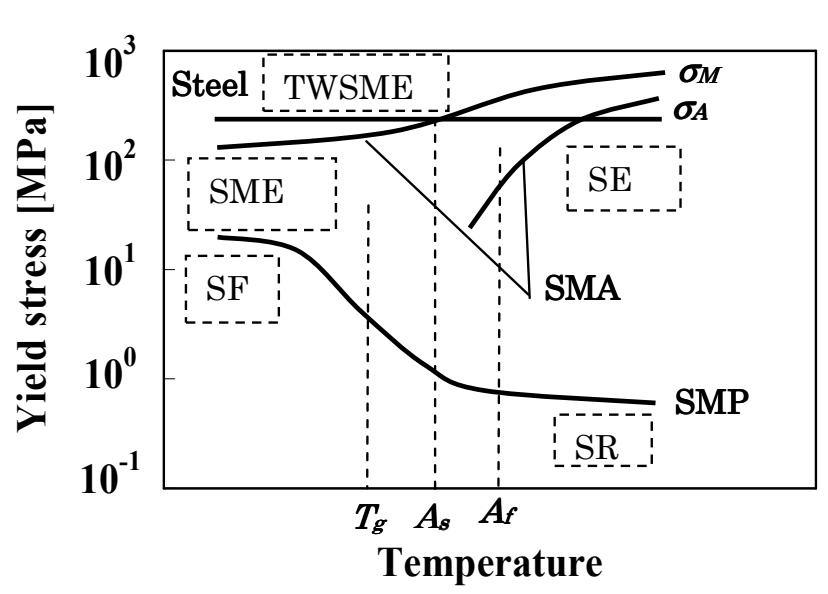

(b) Yield stress

\section{Fig.1 Dependence of elastic modulus and yield stress on temperature for SMA, SMP and steel}

\section{Fabrication of SMC belt with two-way movement}

\section{Materials}

With respect to SMA, two kinds of SMA tapes showing the SME and SE at room temperature were used. The SMA tape showing the SME (SME-SMA tape) was a TiNi SMA tape with width of 5 $\mathrm{mm}$ and thickness of $0.25 \mathrm{~mm}$. The SMA tape showing SE (SE-SMA tape) was a TiNi SMA tape with width of $2.5 \mathrm{~mm}$ and thickness of $0.3 \mathrm{~mm}$. In the shape memory processing, each SMA tape was set along the inside of a fixing ring with inner diameter of $16 \mathrm{~mm}$ and was heat-treated to memorize the round shape with outside diameter of $16 \mathrm{~mm}$. The temperature $A_{f}$ of the SME-SMA tape was $347 \mathrm{~K}$ and that of the SE-SMA tape was $317 \mathrm{~K}$. 
With respect to SMP, polyurethane SMP sheet (MM6520) produced by Mitsubishi Heavy Industries, Ltd. was used. Thickness was $0.25 \mathrm{~mm}$ and the glass transition temperature $T_{g}$ was $338 \mathrm{~K}$.

\section{Structure and deformation properties of SMC belt}

The SMC belt with length of $60 \mathrm{~mm}$, width of $10 \mathrm{~mm}$ and thickness of $0.75 \mathrm{~mm}$ was fabricated by using two kinds of SMA tapes and SMP sheet. In the fabricated SMC belt, the SMP sheet was used as a matrix and the SMA tape as a fiber. Length, width and thickness of the SME-SMA tape were 50 $\mathrm{mm}, 5 \mathrm{~mm}$ and $0.25 \mathrm{~mm}$, respectively. Length, width and thickness of the SE-SMA tape were $50 \mathrm{~mm}$, $2.5 \mathrm{~mm}$ and $0.3 \mathrm{~mm}$, respectively. Two kinds of SMA tapes were located in the central part of the SMC belt.

The structure of the SMC belt is shown in Fig. 2. As can be seen in Fig. 2, the two kinds of shape-memorized round SMA tapes were arranged facing in the opposite directions. The principle of two-way bending behavior in the SMC belt during heating and cooling is shown in Fig. 3. As can be seen in Fig. 3, the SMC belt bends to convex downward (in the direction of the memorized round shape of the SE-SMA tape) by recovery force of the SE-SMA tape at low temperature. It bends to convex upward (in the direction of the memorized round shape of the SME-SMA tape) by recovery force of the SME-SMA tape at high temperature.

\section{Fabrication of SMC belt}

At first, two cuts per tooth were given to one SMP sheet and two kinds of SMA tapes (SME-SMA tape and SE-SMA tape) were passed through these cuts. In this process, the SME-SMA tape and SE-SMA tape were arranged facing in the opposite directions for the memorized round shape. The SMP sheet passed through two kinds of SMA tapes was sandwiched between two SMP sheets from upper and lower sides. The combined material was set in the mold for fabricating the SMC belt. It was confirmed that the SMC belt without bubble and gap among the materials could be fabricated by the following conditions. The upper and lower molds were fastened through the bolts by torque of $6.78 \mathrm{~N} \cdot \mathrm{m}$. The mold was held in the furnace at $448 \mathrm{~K}$ for $30 \mathrm{~min}$ followed by cooling in air.

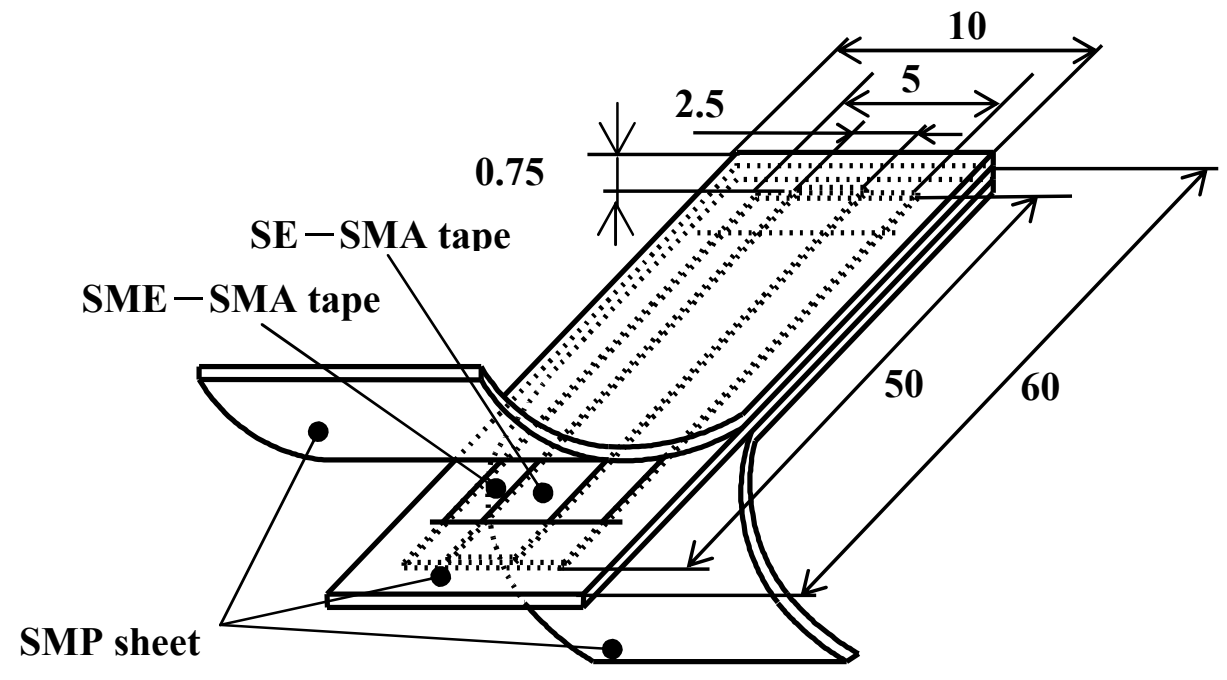

Fig. 2 Structure of SMC belt composed of SME-SMA tape, SE-SMA tape and SMP sheet

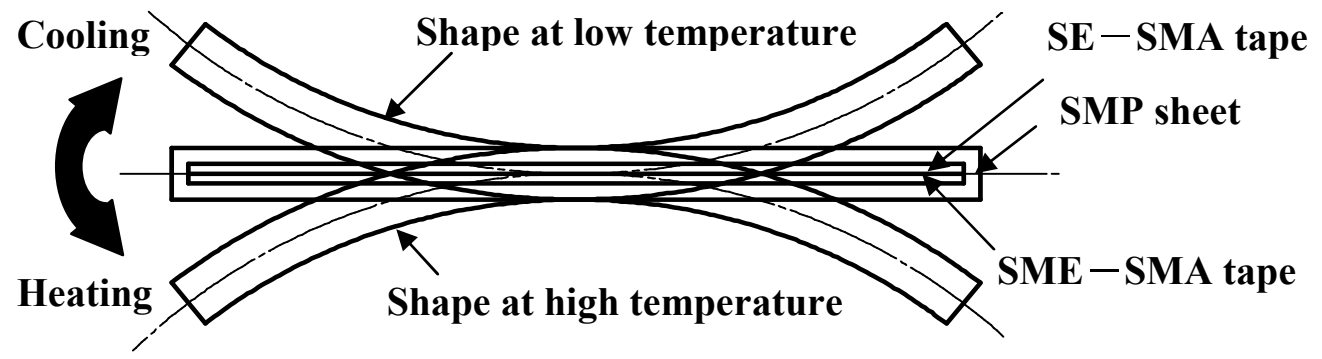

Fig. 3 Principle of two-way bending behavior in SMC belt during heating and cooling 


\section{Two-way bending deformation of SMC belt}

\section{Two-way deformation behavior}

The photograph of the two-way bending deformation of the fabricated SMC belt during heating and cooling is shown in Fig. 4. The heating and cooling were carried out between $293 \mathrm{~K}$ and $363 \mathrm{~K}$. At $293 \mathrm{~K}$, force induced in the SE-SMA tape is high, and therefore the SMC belt bends in the direction of the shape-memorized round shape of the SE-SMA tape. If the SMC belt is heated, the SMP becomes soft and recovery force in the SME-SMA tape increases, and therefore the SMC belt bends in the direction of the shape-memorized round shape of the SME-SMA tape, resulting in the flat plane at $363 \mathrm{~K}$. If the SMC belt is cooled thereafter, recovery force in the SME-SMA tape decreases and recovery force in the SE-SMA tape becomes higher. Therefore, the SMC belt bends again. The fabricated SMC belt bends in two directions by an angle of $56^{\circ}$ during heating and cooling.

\section{Evaluation of deflection based on internal bending moment}

The two-way bending deformation due to variation in temperature appears based on internal bending moment induced in the SE-SMA tape, SME-SMA tape and SMP sheet of the SMC belt. The internal bending moment is proportional to bending rigidity of each element and its dependence on temperature can be evaluated as follows. The bending rigidity of the strip is expressed by $E I$ where $E$ denotes elastic modulus and $I$ second moment of area. If width of cross-section in the strip is denoted by $b$ and height by $h, I=b h^{3} / 12$. The bending rigidity of the SMC belt $E_{c} I_{c}$ is given by the sum of bending rigidity in each element as follows

$$
E_{C} I_{C}=E_{S E} I_{S E}+E_{S M E} I_{S M E}+E_{P} I_{P}
$$

where $E_{c}, E_{S E}, E_{S M E}$ and $E_{p}$ denote elastic modulus of the SMC belt, SE-SMA tape, SME-SMA tape and SMP sheet, respectively. $I_{c}, I_{S E}, I_{S M E}$ and $I_{p}$ represent second moment of area of the SMC belt, SE-SMA tape, SME-SMA tape and SMP sheet, respectively. There is a following relation

$$
I_{C}=I_{S E}+I_{S M E}+I_{P}
$$

The bending rigidity of the SMC belt against external load is given by the sum of bending rigidity in each element as expressed by Eq. (1). However, in the case of the phase transformation by heating and cooling under no load, the internal bending moments in the SMA elements, which are proportional to the bending rigidity of the SE-SMA tape $E_{S E} I_{S E}$ and that of the SME-SMA tape $E_{S M E} I_{S M E}$, act to bend the SMC belt in each direction of the shape-memorized round shape. The internal bending moment in the SMP element, which is proportional to the bending rigidity of the SMP sheet $E_{P} I_{P}$, acts to bend the $\mathrm{SMC}$ belt in the direction of shape-memorized flat shape.

Elastic modulus of the TiNi SMA tape is $70 \mathrm{GPa}$ at temperatures $T>A_{f}$ and $20 \mathrm{GPa}$ at $T<A_{s}$. Therefore, bending rigidity of the SE-SMA tape $E_{S E} I_{S E}$ is $1576 \mathrm{~N} \cdot \mathrm{mm}^{2}$ at temperatures above $293 \mathrm{~K}$. $E_{S M E} I_{S M E}$ of the SME-SMA tape is $520 \mathrm{~N} \cdot \mathrm{mm}^{2}$ at $293 \mathrm{~K}$ and $1842 \mathrm{~N} \cdot \mathrm{mm}^{2}$ at $363 \mathrm{~K}$. Elastic modulus of the SMP sheet is $1 \mathrm{GPa}$ at $T<T_{g}$ and $10 \mathrm{MPa}$ at $T>T_{\mathrm{g}}$. Therefore, bending rigidity of the SMP sheet $E_{p} I_{p}$ is $301 \mathrm{~N} \cdot \mathrm{mm}^{2}$ at $293 \mathrm{~K}$ and $3 \mathrm{~N} \cdot \mathrm{mm}^{2}$ at $363 \mathrm{~K}$. Based on the internal bending moment, the SMC belt deforms as follows. At $293 \mathrm{~K}$ which corresponds to the state of the fabricated SMC belt taken out from the mold, the SMC belt bends in the direction of the shape-memorized round shape of the SE-SMA tape in which internal bending moment is largest. At $363 \mathrm{~K}$, the SMC belt bends in the direction of the shape-memorized round shape of the SME-SMA tape in which internal bending moment is largest. 

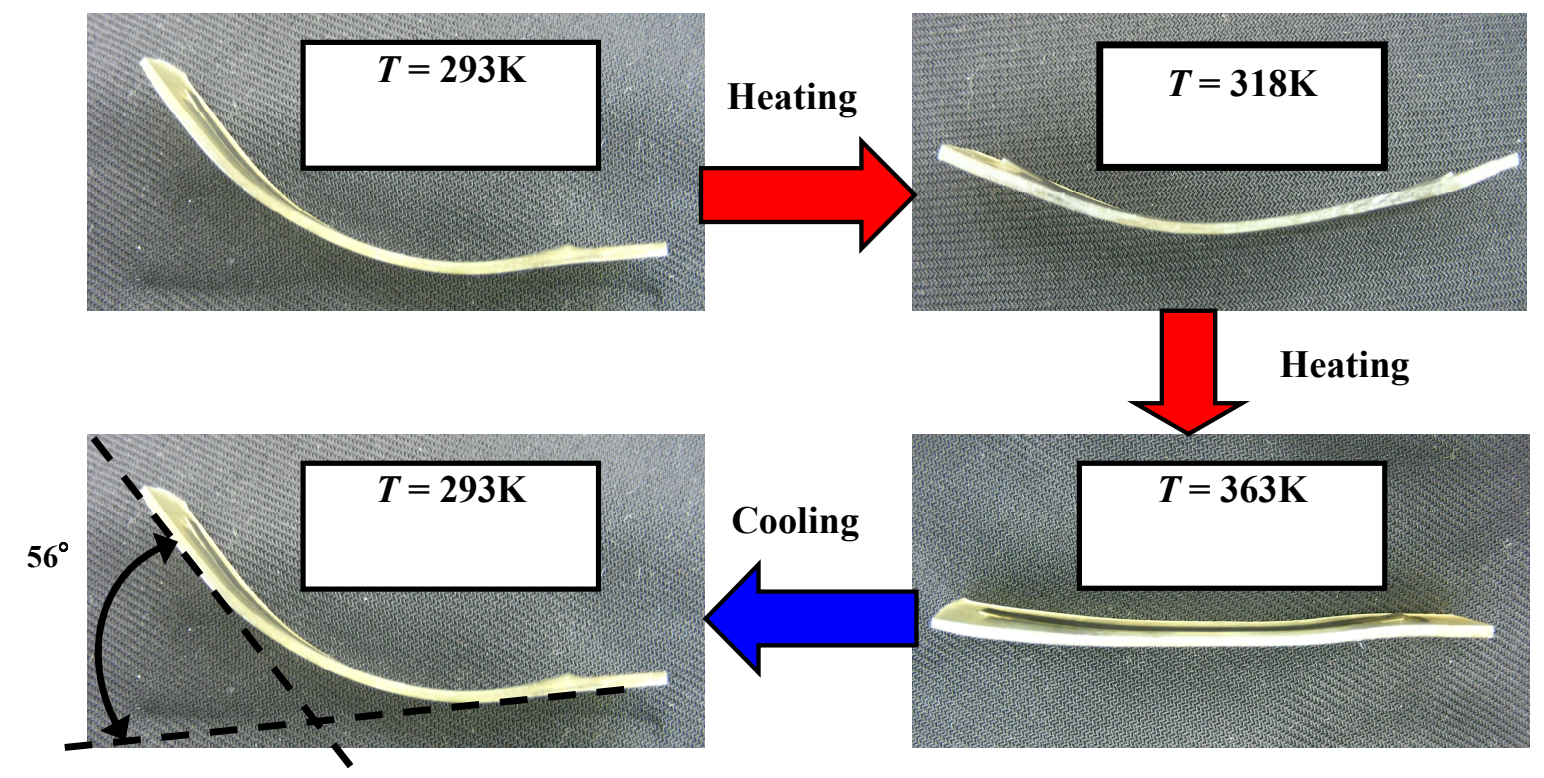

Fig. 4 Photograph of the two-way bending deformation of the SMC belt during heating and cooling

\section{Two-way recovery force of SMC belt in bending}

Recovery force test of the SMC belt was carried out by keeping the bent form in the three-point bending test system. Heating and cooling rates in the furnace in the system were about $1 \mathrm{~K} / \mathrm{min}$ between $293 \mathrm{~K}$ and $363 \mathrm{~K}$. Recovery force which appears when the SMC belt tends to be flat plane during the heating process and that appearing when the SMC belt tends to recover the original bent shape during the cooling process were measured.

The relationship between recovery force and temperature obtained by the three-point bending test for the SMC belt is shown in Fig. 5. The symbols $A_{f, S E}, A_{f, S M E}$ and $T_{g}$ shown in Fig. 5 represent the reverse-transformation finish temperature of the SE-SMA tape, SME-SMA tape and the glass transition temperature of the SMP sheet, respectively. As can be seen in Fig. 5, the behavior of recovery force is different between the heating process and cooling process and the curve draws a hysteresis loop. In the heating process, since temperatures around the $A_{f, S E}$ of the SE-SMA tape are lower than $T_{g}$ of the SMP sheet, recovery force of the SMC is small. Although elastic modulus of the SMP decreases at temperatures around $T_{g}$ of the SMP, recovery force of the SME-SMA tape is smaller than that of the SE-SMA tape, and therefore recovery force of the SMC belt is almost the same as that around $A_{f, S E}$. Recovery force starts to increase at temperatures around $A_{f, S M E}$ of the SME-SMA tape. Since recovery force of the SME-SMA tape becomes larger than that of the SE-SMA tape at temperatures around $A_{f, S M E}$, recovery force of the SMC belt appears.

Recovery force $W$ at high temperature can be evaluated by using deflection $y$ at the center of the SMC belt in the three-point bending for span $l$ by the following equation

$$
W=\frac{48 E_{c} I_{c}}{l^{3}} y
$$

Internal bending moment which appears in the SMC belt with the bent form at $363 \mathrm{~K}$ is estimated as follows. Although internal bending moment in the SME-SMA tape and the SMP sheet acts in the direction where the SMC belt returns to the flat plane, internal bending moment in the SE-SMA tape acts in the opposite direction in which deflection increases. Therefore, from Eq. (1), bending rigidity of the SMC belt is obtained as $E_{c} I_{c}=251 \mathrm{~N} \cdot \mathrm{mm}^{2}$. By substituting the measured values of $l=25.9 \mathrm{~mm}$ and $y=3.4 \mathrm{~mm}$ in Eq. (3), recovery force $W=2.4 \mathrm{~N}$. This value is $69 \%$ of recovery force of $3.5 \mathrm{~N}$ at $363 \mathrm{~K}$. Therefore, recovery force of the SMC belt can be roughly evaluated by Eq. (3). 
In the cooling process, at temperatures around $A_{f, S M E}$ of the SME-SMA tape, since recovery force of the SME-SMA tape is larger than that of the SE-SMA tape, recovery force of the SMC belt is high. At temperatures around $A_{f, S E}$ of the SE-SMA tape, though elastic modulus of the SMP becomes large at temperatures below $T_{g}$, recovery force of the SMC belt is $1.5 \mathrm{~N}$ since recovery force of the SME-SMA tape is still larger than that of the SE-SMA tape.

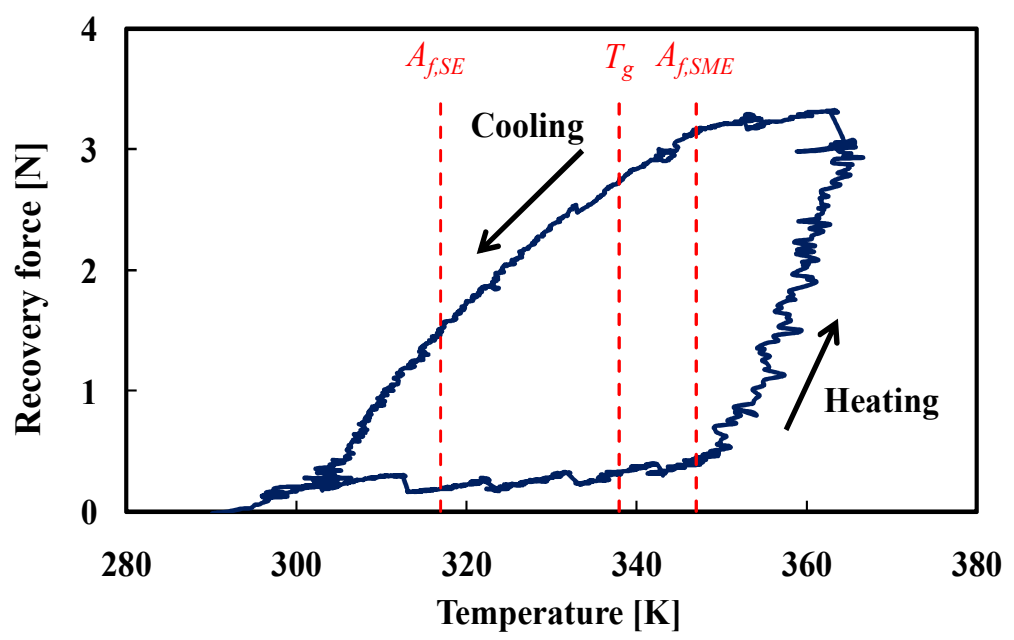

Fig. 5 Relationship between recovery force and temperature during heating and cooling in the three-point bending test for the SMC belt

\section{Conclusions}

The SMC belt composed of two kinds of SMAs and SMP was fabricated and the two-way deformation and recovery force in bending were investigated. The results obtained can be summarized as follows.

(1) Two kinds of SMA tapes showing the SME and SE were heat-treated to memorize the round shape, respectively. The shape-memorized round SMA tapes were sandwiched by one SMP sheet in the central part and by two SMP sheets from upper and lower sides. The SMC belt was fabricated without bubble and gap by using the appropriate factors for the number of SMP sheets, pressing force, heating temperature and holding time.

(2) The two-way bending deformation with an angle of 56 degrees was observed.

(3) Based on the two-way characteristics of the SMC belt, recovery force increased during heating and decreased during cooling.

\section{References}

[1] Otsuka, K. and Waymam, C. M., eds., Shape Memory Materials, Cambridge University Press, (1998), pp. 1-49.

[2] Hayashi, S., Properties and Applications of Polyurethane Series Shape Memory Polymer, Int. Progr. Urethanes, 6 (1993), pp. 90-115.

[3] Tobushi, H., Hayashi, S., Hoshio, K., Makino, Y. and Miwa, N., Bending Actuation Characteristics of Shape Memory Composite with SMA and SMP, J. Intell. Mater. Syst. Struct., 17 (2006), pp. 1075-1081. 